\title{
PULSAR INTERFEROMETRY WITH MICROARCSECOND RESOLUTION
}

\author{
A. Wolszczan \\ NAIC, Arecibo Observatory, Arecibo, PR 00613 \\ J.M. Cordes \\ Astronomy Department and NAIC, Cornell University, \\ Ithaca, NY 14853
}

\begin{abstract}
An 'interstellar interferometer' related to a double imaging of the pulsar PSR $1237+25$ by refraction in the interstellar medium has been used to resolve the pulsar magnetosphere for the first time. With an interferometer baseline $\sim 1 A U$, we have measured a typical transverse separation between the emitting regions of PSR $1237+25$ to be $\sim 10^{8} \mathrm{~cm}$, or an angular separation $\sim 0.02 \mu$ as.
\end{abstract}

Refractive effects can significantly modify the interstellar scintillation patterns arising from diffractive scatter of pulsar signals propagating through the interstellar medium (e.g. Hewish et al. 1985; Cordes and Wolszczan 1986). Strong refraction events sometimes split the apparent pulsar image into two or more distinct subimages. The resultant dynamic spectra (intensity as a function of time and frequency) are the superposition of separate patterns from each subimage combined with oscillatory cross terms caused by beating between subimages. The oscillatory terms are equivalent to fringes obtained with an interferometer baseline $\sim 1 \mathrm{AU}$, sufficiently large to potentially resolve the pulsar magnetosphere (Cordes et al. 1986).

The dynamic spectra of PSR $1237+25$ observed in December 1986 at 430 $\mathrm{MHz}$ with the Arecibo radiotelescope and with the 3-level, $40 \mathrm{MHz}$ correlation spectrometer are shown in Fig. 1a. They display a strikingly periodic intensity modulation as a function of time and frequency. A model to explain this modulation (Wolszczan and Cordes 1987 and references therein) consists of a thin screen in which refractive phase variations are strong enough to give rise to a pair of diffracted subimages of the pulsar. Beating between subimages leads to oscillations (fringes) in the dynamic spectra, best characterized by the oscillation period in frequency, $P_{\nu}$. The transverse displacement of a source by a distance $\delta x_{s}$ shifts the observed fringes by approximately $\delta \phi \approx 3 \cdot 10^{-5} \nu \delta x_{s}\left[\left(D / D_{s}-1\right) / D P_{\nu}\right]^{1 / 2}$ radians, where $\nu$ is the observing frequency, $\mathrm{D}$ is the pulsar distance and $D_{s}$ is the distance between the pulsar and the screen. Fringe shifts may arise, if different pulse components are emitted from locations that are separated in the transverse direction (e.g. Cordes et al. 1983). We have resolved such separations by detecting fringe phase shifts in the dynamic spectra of PSR $1237+25$ at different pulse longitudes (one pulsar period equals $360^{\circ}$ longitude) as shown in Fig. 1b,c. The range of spatial separations of the emission regions calculated for $D=2 D_{s}$ is indicated on the right hand side of Fig. 1c. For a dipolar magnetic field and emission at a constant altitude, 285

M. J. Reid and J. M. Moran (eds.), The Impact of VLBI on Astrophysics and Geophysics, 285-286.

(c) 1988 by the IAU. 
the fringe phase should be symmetric with respect to the pulse centroid and it should monotonically increase through the origin. This is in contradiction to the measurements, suggesting that the magnetic field of PSR $1237+25$ may deviate strongly from a dipolar form in the emission region and emission altitudes may vary such that transverse separations are much larger for a given pulse longitude than implied by the dipole model. Alternatively, the refracting screen may be located much nearer the pulsar than the Earth $\left(D \gg D_{s}\right)$, which would lead to transverse separations much smaller than for the screen that is midway $\left(D=2 D_{s}\right)$.
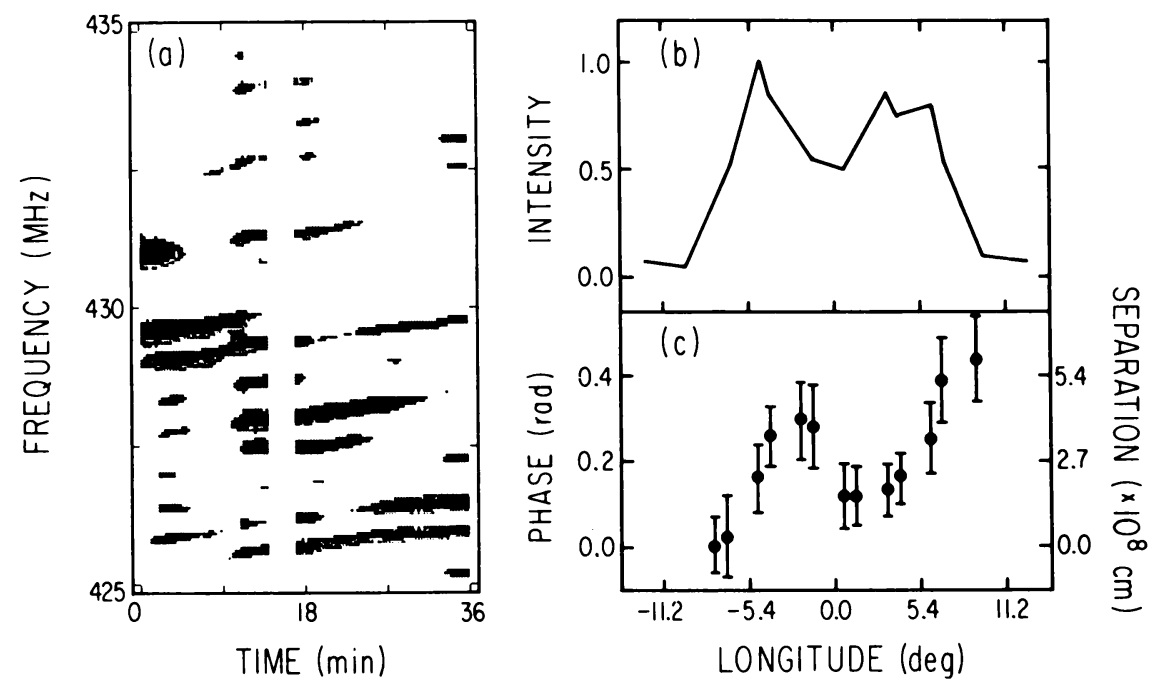

Fig. 1. PSR 1237+25: (a) Dynamic spectrum, (b) integrated pulse profile and $(c)$ fringe phase as a function of pulse longitude.

In addition to resolving the pulsar magnetosphere, our measurements suggest that a discrete refracting structure of size $>1 \mathrm{AU}$ crossed the line of sight, producing a predominantly double image of the pulsar with a splitting angle between subimages of about 3.3 mas. This episode may be similar in origin to recent caustic events seen in the light curves of compact extragalactic radio sources (Fiedler et al. 1987).

This work was supported by the Alfred P. Sloan Foundation, by the National Science Foundation through grant 85-20530 to Cornell University, and by the National Astronomy and Ionosphere Center at Cornell which operates the Arecibo Observatory under contract to the National Science Foundation.

\section{REFERENCES}

Cordes, J.M., Weisberg, J.M., and Boriakoff, V. 1983, Ap.J., 268, 370.

Cordes, J.M., and Wolszczan, A. 1986, Ap. J. (Letters) , 307, L27.

Cordes, J.M., Pidwerbetsky, A., and Lovelace, R.V.E. 1986, Ap. J., 310, 737.

Fiedler,R.L., Dennison,B., Johnston,K.J., and Hewish,A. 1987, Nature, , in press.

Hewish, A., Wolszczan, A., and Graham, D. A. 1985, M.N.R.A.S., 192, 799.

Wolszczan, A., and Cordes, J. M. 1987, Ap. J. (Letters), , submitted. 